\title{
Hitting times for random walks on vertex-transitive graphs
}

\author{
By DAVID ALDOUS \\ Department of Statistics, University of California, Berkeley CA 94720, U.S.A.
}

\author{
(Received 4 July 1988)
}

\begin{abstract}
For random walks on finite graphs, we record some equalities, inequalities and limit theorems (as the size of graph tends to infinity) which hold for vertex-transitive graphs but not for general regular graphs. The main result is a sharp condition for asymptotic exponentiality of the hitting time to a single vertex. Another result is a lower bound for the coefficient of variation of hitting times. Proofs exploit the complete monotonicity properties of the associated continuous-time walk.
\end{abstract}

\section{Introduction}

Random walks on graphs have been studied in a wide variety of contexts. On highly-symmetric (e.g. distance-transitive) graphs it is feasible to attempt analytic calculations of $n$-step transition probabilities and exact hitting time distributions: see $[10,16,18]$. At the other extreme, for general graphs there are various general bounds known $[5,1,4]$ and in the more general setting of reversible Markov chains there are techniques for obtaining long-range estimates [20].

Let $G=(V, \mathscr{E})$ be a finite connected regular graph, of degree $r \geqslant 2$. Random walk on $G$ is the discrete-time Markov chain with transition matrix $P$ of the form

$$
P(v, w)= \begin{cases}1 / r & \text { if }(v, w) \text { is an edge } \\ 0 & \text { if not. }\end{cases}
$$

Regularity implies that $P$ is symmetric and hence the stationary distribution $\pi$ is the uniform distribution on $V$. The graph $G$ is vertex-transitive if its group of automorphisms acts transitively on $V$ (see [7] for a careful account of such symmetry conditions). This is a stronger requirement than regularity. We shall suppose graphs are vertex-transitive except where otherwise stated.

Write $\mathbb{E}$ for expectation, var for variance and $\mathscr{L}$ for distribution : subscripts $v$ or $\pi$, e.g. in $\mathbb{E}_{v}, \mathbb{E}_{\pi}$, indicate that the walk starts at $v$ or with the uniform distribution. Write $T_{w}$ for the first hitting time on a vertex $w$. Thus $\mathscr{L}_{v} T_{w}$ denotes the distribution of the time for the random walk started at $v$ to first hit $w$. Write $X(n)$ for the position of the walk at time $n$.

The classical matrix approach to Markov chains yields expressions for mean hitting times and related quantities, and these may be specialized to the setting of random walks on graphs: see $[13,11]$. The only paper known to the author which deals with precisely vertex-transitive graphs is [19] which uses matrix methods to obtain e.g. the expectation and variance assertions of Proposition 2 below and exhibits numerical calculations for a particular graph, the triangular prism. 
We first list equalities and inequalities for random walks on vertex-transitive graphs. We shall show, or at least remark, later that these results are not true in general for regular graphs. Some of the results, e.g. Proposition 1, are obvious and are recorded for completeness.

Proposition 1. $\mathscr{L}_{\pi} T_{v}$, and hence $\mathbb{E}_{\pi} T_{v}$, does not depend on $v$.

The quantity $\mathbb{E}_{n} T_{v}$ plays a large role in this paper: let us denote it by $\alpha$ to emphasize that it is independent of $v$.

Proposition 2. For each pair $(v, w)$ we have $\mathscr{L}_{v} T_{w}=\mathscr{L}_{w} T_{v}$ and so in particular $\mathbb{E}_{v} T_{w}=\mathbb{E}_{w} T_{v}$ and $\operatorname{var}_{v} T_{w}=\operatorname{var}_{w} T_{v}$.

Proposirion 3. Given a distinct pair $(v, w)$ define $T=\min \left(T_{v}, T_{w}\right)$ to be the first time that the walk hits $v$ or $w$. Then

$$
P_{\pi}(X(T)=v)=P_{\pi}(X(T)=w)=\frac{1}{2}
$$

and moreover

$$
P_{n}(T=n, X(T)=v)=P_{n}(T=n, X(T)=w) \text { for all } n \geqslant 0 .
$$

Proposition 4. $\mathbb{E}_{v} T_{w} \leqslant 2 \alpha$ for all $(v, w)$.

Proposition 5. $\mathbb{E}_{v} T_{w} \geqslant \frac{1}{2}|V|$ for all $v \neq w$.

Proposition 6. For all $v \neq w$

$$
\frac{\operatorname{var}_{v} T_{w}}{\left(\mathbb{E}_{v} T_{w}\right)^{2}} \geqslant c-\frac{1}{\mathbb{E}_{v} T_{w}}
$$

where $c=(e-2) /(e-1)>0 \cdot 4$.

Proposition 6 gives a lower bound for the 'coefficient of variation' of first passage time distributions. Some motivation for this result is described in Section 5 .

Because the transition matrix $P$ is symmetric, it has real eigenvalues

$$
1=\lambda_{1}^{\prime}>\lambda_{2}^{\prime} \geqslant \lambda_{3}^{\prime} \ldots
$$

For $k \geqslant 2$ write $\tau_{k}=1 /\left(1-\lambda_{k}^{\prime}\right)$ and call $\tau_{2}$ the relaxation time. The mean hitting time $\alpha$ can be expressed in terms of the eigenvalues via

$$
\alpha=\sum_{k=2}^{|V|} \tau_{k}
$$

(see Section 3).

Our final results concern sequences $G_{K}$ of vertex-transitive graphs. Here quantities such as $\alpha$ and $\tau_{2}$ depend on $K$, but we shall not write the $K$ explicitly. All limits are as $K$ tends to infinity. Write $\mu_{1}$ for the exponential distribution with mean 1 . Convergence of distributions means, of course, 'convergence in distribution'.

Proposition 7. For a sequence of verlex-transitive graphs with $|\boldsymbol{T}| \rightarrow \infty$, the following are equivalent:

(a) $\tau_{2} / \alpha \rightarrow 0$;

(b) $\mathscr{L}_{n}\left(T_{w} / \alpha\right) \rightarrow \mu_{1}$ and $\operatorname{var}_{n}\left(T_{w} / \alpha\right) \rightarrow 1$. 
Note that, by Proposition $5, \alpha=\Omega(|V|)$ and so condition $(a)$ is implied by

$$
\tau_{2} /|V| \rightarrow 0 \text {. }
$$

Informally, condition (3) is 'only just' stronger than condition $(a)$.

Proposition 8. Consider a sequence of vertex-transitive graphs with $|\bigvee| \rightarrow \infty$ and satisfying condition (a) of Proposition 7. Then

(c) $\max _{v, w}\left(\mathbb{E}_{v} T_{w} / \alpha\right) \rightarrow 1$.

(d) Suppose $v=v_{K}, w=w_{K}$ are such that $\mathbb{E}_{v} T_{w} / \alpha \rightarrow \theta$. Then

$$
\mathscr{L}_{v}\left(T_{w} / \alpha\right) \rightarrow \theta \mu_{1}+(1-\theta) \delta_{0}
$$

where $\delta_{0}$ is the distribution degenerate at $\mathbf{0 .}$

Section 4 contains discussion of Propositions 7 and 8 , and a version of assertion (d) for $L$-tuples. Results similar in spirit to Propositions 7 and 8 hold for very general Markov processes; but the setting of random walks on vertex-transitive graphs permits cleaner statements and proofs.

This ends the list of results. Loosely speaking, we shall see in Section 2 that everything is obvious from classical techniques except for the final three Propositions. In Section 3 we discuss complete monotonicity, and in Sections 4 and 5 use it to prove the remaining results.

\section{Easy results}

Proposition 1 genuinely is 'obvious by symmetry': considering an automorphism $\gamma$ taking $v$ to $w$ does lead to the conclusion $\mathscr{L}_{n} T_{v}=\mathscr{L}_{n} T_{w}$. Proposition 2 is similarly obvious under the hypothesis

for each pair $v, w \in V$ there exists an automorphism $\gamma$

$$
\text { such that } \gamma(v)=\gamma(w) \text { and } \gamma(w)=\gamma(v) \text {. }
$$

But (4) turns out to be strictly stronger than vertex-transitivity, so we resort to the analytic argument below.

Proof of Proposition 2. For Markov chains there is the following classical relationship between the generating functions of the $n$-step transition probabilities and the generating functions of the hitting times:

$$
g_{v w}(z)=h_{v w}(z) / h_{w w}(z),
$$

where

$$
h_{v w}(z)=\sum_{n=0}^{\infty} P_{v}(X(n)=w) z^{n}, \quad g_{v w}(z)=\sum_{n=0}^{\infty} P_{v}\left(T_{w}=n\right) z^{n} .
$$

On any regular graph, the symmetry of $P$ implies $h_{v w}=h_{w v}$. On a vertex-transitive graph we have

$$
P_{v}(X(n)=v)=P_{w}(X(n)=w) \text { for all } v, w \in V, n \geqslant 0
$$

and hence $h_{v w}=h_{w v}$. Then (5) implies $g_{v w}=g_{w v}$ and hence $\mathscr{L}_{v} T_{w}=\mathscr{L}_{w} T_{v}$.

This argument shows that on a graph the condition

$$
\mathscr{L}_{v} T_{w}=\mathscr{L}_{w} T_{v} \quad \text { for all } v, w \in V
$$

is equivalent to $(6)$. 
Problem 9. If random walk on a graph satisfies (6), is the graph necessarily vertextransitive?

Proof of Proposition 4. For any $v, w, x$

$$
\mathbb{E}_{v} T_{w} \leqslant \mathbb{E}_{v} T_{x}+\mathbb{E}_{x} T_{w}=\mathbb{E}_{x} T_{v}+\mathbb{E}_{x} T_{w}
$$

by Proposition 2. Averaging over $x$ gives

$$
\mathbb{E}_{v} T_{w} \leqslant \mathbb{E}_{n} T_{v}+\mathbb{E}_{n} T_{w}=2 \alpha .
$$

Proof of Proposition 5. Let $T_{v}^{+} \geqslant 1$ be the first return time to $v$. Markov chain theory says $\mathbb{E}_{v} T_{v}^{+}=1 /(\pi(v))=|V|$ in our setting. So if $w \neq v$ then

$$
|V|=\mathbb{E}_{v} T_{v}^{+} \leqslant \mathbb{E}_{v} T_{w}+\mathbb{E}_{w} T_{v}=2 \mathbb{E}_{v} T_{w}
$$

by Proposition 2 .

Proof of Proposition 3. This is a variation on the classical proof of (5). By conditioning on $T_{v}$,

$$
P_{\pi}\left(X(n)=v, T_{w}>n\right)=\sum_{m=0}^{n} P_{n}\left(T_{v}=m, T_{w}>m\right) P_{v}\left(X(n-m)=v, T_{w}>n-m\right) .
$$

This leads to the generating function relationship

$$
a_{v w}(z)=b_{v w}(z) c_{v w}(z)
$$

where

$$
\begin{aligned}
& a_{v w}(z)=\sum_{n=0}^{\infty} P_{n}\left(X(n)=v, T_{w}>n\right) z^{n}, \\
& b_{v w}(z)=\sum_{n=0}^{\infty} P_{n}\left(T_{v}=n, T_{w}>n\right) z^{n}, \\
& c_{v w}(z)=\sum_{n=0}^{\infty} P_{v}\left(X(n)=v, T_{w}>n\right) z^{n} .
\end{aligned}
$$

To establish the proposition it suffices to show that $b_{v w}$ is symmetric in $(v, w)$. By (7) it is enough to show that $a_{v w}$ and $c_{v w}$ are symmetric in $(v, w)$.

For $0 \leqslant m \leqslant n$,

$$
P_{v}\left(X(n)=v, T_{w}=m\right)=P_{v}\left(T_{w}=m\right) P_{w}(X(n-m)=v)
$$

and this is symmetric in $(v, w)$ by Proposition 2 and the symmetry of $P$. Summing over $m$ we see that $P_{v}\left(X(n)=v, T_{w} \leqslant n\right)$ is symmetric in $(v, w)$. It follows that $P_{v}\left(X(n)=v, T_{w}>n\right)$ is symmetric, and hence $c_{v w}$ is symmetric. The same argument, with $P_{n}$ in place of $P_{v}$, shows that $a_{v w}$ is symmetric.

Remarks. Let us briefly indicate a way to produce counter-examples to these Propositions for regular graphs. Suppose $(v, w)$ is an edge in a graph $G$ such that removing this edge splits the graphs into two components, say $v \in C_{1}, w \in C_{2}$. It is easy to show (see [5]) that

$$
\mathbb{E}_{v} T_{w}=2\left|C_{1}\right|+1, \quad \mathbb{E}_{w} T_{v}=2\left|C_{2}\right|+1 .
$$

We can construct such an edge in a 3-regular graph $G$ where $\left|C_{1}\right|=5$ and where $\left|C_{2}\right|$ is arbitrarily large. Thus (ef. Proposition 2) the ratio $\mathbb{E}_{w} T_{v} / \mathbb{E}_{v} T_{w}$ may be arbitrarily 
large. Moreover $\pi\left(C_{2}\right)$ is arbitrarily close to 1 , so (cf. Proposition 1) it is easy to see that the ratio $\mathbb{E}_{n} T_{v} / \mathbb{E}_{n} T_{w}$ may be arbitrarily large, and (cf. Proposition 3 ) that $P_{n}\left(T_{w} \leqslant T_{v}\right)$ may be arbitrarily close to 1 . Moreover (cf. Proposition 5) $\mathbb{E}_{v} T_{w} /|V|$ may be arbitrarily small.

Somewhat more complicated examples (ladders attached to expanders: see [4]) show that for regular graphs the ratio $\mathbb{E}_{v} T_{w} / \mathbb{E}_{n} T_{w}$ may be arbitrarily large (cf. Proposition 4).

\section{Complete monotonicity and continuous-time reversible chains}

A function $f(t)$ defined for $t \geqslant 0$ is completely monotone if

$$
f(t)=\int_{0}^{\infty} e^{-t x} \beta(d x)
$$

for some positive measure $\beta$ on $[0, \infty)$. A probability distribution $\nu$ on $[0, \infty)$ is completely monotone if it satisfies the following equivalent conditions:

(a) $t \rightarrow \nu(t, \infty)$ is a completely monotone function;

(b) $\nu$ is a mixture of the distribution $\delta_{0}$ degenerate at 0 and some distribution on $(0, \infty)$ with completely monotone density function;

(c) $\nu$ is the distribution of $\xi \dot{V}$, where $\xi$ and $V$ are independent, $\xi$ has the exponential(1) distribution $\mu_{1}$, and $0 \leqslant V<\infty$.

Write CM for the set of completely monotone distributions. The following result is straightforward; since it is central to our later arguments, let us sketch the proof.

Lemma 10. For $1 \leqslant k<\infty$, let $\nu_{k} \in C M$.

(a) If $\nu_{k} \rightarrow \nu$ then $\nu \in C M$.

(b) Suppose each $\nu_{k}$ has mean 1, and suppose var $\left(\nu_{k}\right) \rightarrow 1$. Then $\nu_{k} \rightarrow \mu_{1}$.

Proof. Use form $(c)$ of the definition of CM. By passing to a subsequence, we may suppose that $\nu_{k}=\mathscr{L}\left(\xi_{k} V_{k}\right)$ and $\left(\xi_{k}, V_{k}\right) \stackrel{d}{\rightarrow}(\xi, V)$ say, and then $\xi$ and $V$ must be independent. Thus $\nu=\mathscr{L}(\xi V) \in \mathrm{CM}$. In setting $(b)$,

$$
\operatorname{var}\left(\nu_{k}\right)=\operatorname{var}\left(\xi_{k} V_{k}\right)=1+2 \operatorname{var}\left(V_{k}\right) \rightarrow 1 \text {. }
$$

So $\operatorname{var}\left(V_{k}\right) \rightarrow 0$ and so $V_{k} \stackrel{d}{\rightarrow} 1$. So $\xi_{k} V_{k} \stackrel{d}{\rightarrow} \xi$, as required.

A Markov transition matrix $P$ can be used to define a continuous-time Markov chain $X(t)$ for $t \geqslant 0$ via

$$
P(X(t+\delta)=j \mid X(t)=i) \sim \delta P_{i j} \quad \text { as } \delta \rightarrow 0 .
$$

Equivalently, the continuous-time chain can be derived from the discrete-time chain by replacing the deterministic (unit time) interval between jumps with a random (exponential, mean 1) interval. So in particular we can talk about continuous-time random walks on graphs. It is usually easy to transfer results from one setting to the other. In particular

$$
\begin{aligned}
& \mathbb{E}_{v} T_{w} \text { is the same in discrete or continuous time; } \\
& \text { (continuous-) } \operatorname{var}_{v} T_{w}=\left(\text { discrete }- \text { ) } \operatorname{var}_{v} T_{w}+\mathbb{E}_{v} T_{w} .\right.
\end{aligned}
$$

The Propositions already proved are identical in the two settings. We now switch 
to the continuous-time setting; the relations above show that conclusions of later Propositions can be applied back in the discrete-time setting.

Continuous-time random walks on graphs are a special case of continuous-time reversible Markov chains. Basic properties of such chains are discussed in [14], which contains the following two lemmas. Write $T_{A}$ for the first hitting time on a set $A$ of states.

Leмma 11. For a continuous-time reversible Markov chain, and for any set $A$ of states, $\mathscr{L}_{\pi}\left(T_{A}\right) \in C M$. Also $\mathscr{L}_{\rho}\left(T_{A}\right) \in C M$, where $\rho$ is the first-exit-place distribution

$$
\rho=\mathscr{L}_{\pi}(X(S)), \quad S=\min \left\{t: X(t) \in A^{c}\right\} .
$$

This complete monotonicity property is the main reason for working in continuous time.

Lemma 12 (spectral representation). For a continuous-time reversible Markov chain with stationary distribution $\pi$,

$$
P_{v}(X(t)=w)=\sqrt{ }(\pi(w) / \pi(v)) \sum_{k=1}^{|V|} e^{-\lambda_{k} t} u_{v k} u_{w k}
$$

where $U=\left(u_{v k}\right)$ is an orthonormal matrix and where, for $P$ symmetric, $\lambda_{k}=1-\lambda_{k}^{\prime}$ in $(1)$.

Here are some relations between hitting times and the spectral representation.

Lemma 13. For a continuous-time Markov chain with stationary distribution $\pi$ let $\lambda_{k}$ for $2 \leqslant k \leqslant|V|$ be as in the spectral representation and let $\tau_{k}=\lambda_{k}^{-1}$. Then

(a) $\Sigma_{w} \pi(w) \mathbb{E}_{\pi} T_{w}=\Sigma_{k=2}^{|V|} \tau_{k}$

(b) if $\pi$ is uniform then

$$
|V|^{-1} \sum_{w} \mathbb{E}_{\pi} T_{w}^{2}=2|V|^{-1} \sum_{w}\left(\mathbb{E}_{\pi} T_{w}\right)^{2}+\frac{1}{2} \sum_{k=2}^{|V|} \tau_{k}^{2} .
$$

Specializing to continuous-time random walks on vertex-transitive graphs, where $\alpha=\mathbb{E}_{n} T_{w}$, we obtain

$$
\begin{gathered}
\alpha=\sum_{k=2}^{|V|} \tau_{k}, \\
\mathbb{E}_{\pi} T_{w}^{2}=2 \alpha^{2}+\frac{1}{2} \sum_{k=2}^{|V|} \tau_{k}^{2} .
\end{gathered}
$$

Formulae of this type are standard, though usually given in settings where more symmetry is present, e.g. [12]. Result (a) is given explicitly in [8]; we end this section with the proof of $(b)$.

Proof of Lemma 13. Fix $j$. We quote some results about general (i.e. not necessarily reversible) finite continuous-time chains.

$$
\begin{aligned}
Z_{i, j} & =\int_{0}^{\infty}\left(P_{i}(X(t)=j)-\pi(j)\right) d t \text { exists; } \\
\mathbb{E}_{i} T_{j} & =\left(Z_{j j}-Z_{i j}\right) / \pi(j) ; \\
\mathbb{E}_{\pi} T_{j} & =Z_{j j} / \pi(j) ; \\
\mathbb{E}_{n} T_{j}^{2} & =2 \sum_{i} \gamma(i) \mathbb{E}_{i} T_{j} ;
\end{aligned}
$$

where $\quad \gamma(i)=\mathbb{E}_{n}\left(\right.$ number of visits to $i$ before $\left.T_{j}\right)=Z_{j j} \pi(i) / \pi(j)-Z_{j i}$. 
Here (11), (12), (14) follow from the classical matrix approach to mean hitting times (see [15]; a direct approach via renewal theory is in [4]). Identity (13) is an 'occupation measure identity' in the sense of [17]; it can be obtained directly by writing $\mathbb{E}_{\pi} T_{j}^{2}=2 \int_{0}^{\infty} \mathbb{E}\left(T_{j}-t\right)^{+} d t$ and conditioning on $X(t)$.

In the setting of our lemma, $\pi$ is uniform and reversibility implies $Z_{i j}=Z_{j i}$. Putting the identities together,

$$
\begin{aligned}
\mathbb{E}_{\pi} T_{j}^{2} & =2|V| \sum_{i}\left(Z_{j j}-Z_{i j}\right)^{2} \\
& =2|V|^{2} Z_{j j}^{2}+2|V| \sum_{i} Z_{i j}^{2} \quad \text { because } \sum_{i} \pi(i) Z_{i j}=0 \\
& =2\left(\mathbb{E}_{\pi} T_{j}\right)^{2}+2|V| \sum_{i} Z_{i j}^{2} .
\end{aligned}
$$

Then, writing $p_{i j}(t)=P_{i}(X(t)=j)$,

$$
\begin{aligned}
\sum_{i} Z_{i j}^{2} & =\sum_{i}\left(\int_{0}^{\infty}\left(p_{i j}(s)-\pi(j)\right) d s\right)\left(\int_{0}^{\infty}\left(p_{i j}(t)-\pi(j)\right) d t\right) \\
& =\iint \sum_{i}\left(p_{i j}(s)-\pi(j)\right)\left(p_{i j}(t)-\pi(j)\right) d s d t \\
& =\int t\left(p_{j j}(2 t)-\pi(j)\right) d t \quad \text { because } \sum_{i} p_{i j}(s) p_{i j}(t)=\sum_{i} p_{j i}(s) p_{i j}(t)=p_{j j}(s+t) \\
& =\int_{k=2}^{|V|} u_{j k}^{2} t e^{-2 \lambda_{k} t} d t \quad \text { by the spectral representation } \\
& =\frac{1}{4} \sum_{k=2}^{|V|} u_{j k}^{2} \lambda_{k}^{-1}=\frac{1}{4} \sum_{k=2}^{|V|} u_{j k}^{2} \tau_{k} .
\end{aligned}
$$

By orthogonality,

$$
\sum_{i} \sum_{j} Z_{i j}^{2}=\frac{1}{4} \sum_{k=2}^{|v|} \tau_{k} .
$$

Now averaging over $j$ in (15) gives the result.

\section{Asymptotic results}

We first discuss, and then prove, Propositions 7 and 8 . There is a general heuristic idea that, for finite Markov chains, if the time to approach stationarity is small compared to the number of states then hitting times on small subsets have approximately exponential distribution. A general, though not easily applicable, formulation is given in [2]. In the particular context of random walks on vertextransitive graphs, our results show that $\tau_{2}=o\left(\mathbb{E}_{n} T_{w}\right)$ is essentially the necessary and sufficient condition for approximate exponentiality. In particular, a sufficient condition is $\tau_{2}=o(|V|)$, which is often very easy to verify. Thus results of [12] concerning card-shuffling processes, and simple random walk on the $d$-dimensional torus $Z_{K}^{d}(d \geqslant 2$ fixed, $K \rightarrow \infty)$ can be derived from our results without extensive calculation. Incidentally, the 2-dimensional torus provides an example where

$$
|V|=K^{2}, \quad \mathbb{E}_{\pi} T_{w} \sim c_{1} K^{2} \log K, \quad \tau_{2} \sim c_{2} K^{2},
$$

so the weaker sufficient condition does not hold although the full necessary and sufficient condition does hold. See [9] for direct treatment of this example. 
So far we have implicitly been considering the case where the walk starts with the uniform distribution. In considering $T_{w}$ for the walk started at $v$, there may be some chance $(\theta$, say) that the walk hits $w$ within time $o(\alpha)$, e.g. if $v$ and $w$ are 'close'. Thus $T_{w} / \alpha$ has chance $\theta$ to be nearly 0 ; Proposition 8 says that the remaining part of the distribution is approximately exponential.

Proof of Proposition 7. With the machinery already established, the proof is easy. From (9), (10),

$$
\operatorname{var}_{n}\left(T_{v} / \alpha\right)-1=\frac{1}{2} \sum_{k=2}^{|V|} \tau_{k}^{2} / \alpha^{2}
$$

Since $\tau_{k} \leqslant \tau_{2}$ for $k \geqslant 2$ we have

$$
\sum_{k=2}^{|V|} \tau_{k}^{2} \leqslant \alpha \tau_{2}
$$

So for a sequence of vertex-transitive graphs $G_{K}, K \rightarrow \infty$, the right-hand side of (16) tends to 0 if and only if $\tau_{2} / \alpha \rightarrow 0$. Moreover if $\tau_{2} / \alpha \rightarrow 0$ then (16), Lemma 10 and Lemma 11 combine to imply $\mathscr{L}_{\pi}\left(T_{w} / \alpha\right) \rightarrow \mu_{1}$. This establishes Proposition 7 .

Proof of Proposition 8. Now suppose the conditions of Proposition 7 hold, so in particular

$$
\mathscr{L}_{\pi}\left(T_{w} / \alpha\right) \rightarrow \mu_{1} .
$$

Note that the distribution $\mathscr{L}_{v} T_{w}$ is not CM (its density at 0 is 0 ), so we cannot directly apply Lemma 10 here. Instead we use an indirect argument exploiting Proposition 7.

Take $v=v_{K}, w=w_{K}$ as in the hypothesis of Proposition 8. Define

$$
T=\min \left(T_{v}, T_{w}\right) .
$$

We assert that (all limits are as $K \rightarrow \infty$ )

$$
\lim \inf \mathbb{E}_{n}(T / \alpha) \geqslant \frac{1}{2} .
$$

By considering subsequences we may suppose $\mathscr{L}_{n}(T / \alpha) \stackrel{d}{\rightarrow} S$, say, and so (using the variance assertion of Proposition 7) $\mathbb{E} S=\lim \mathbb{E}_{n}(T / \alpha)$. Write $f_{v}^{K}, f^{K}$ for the densities of $\mathscr{L}_{\pi}\left(T_{v} / \alpha\right)$ and $\mathscr{L}_{\pi}(T / \alpha)$. Then

$$
f^{K} \leqslant f_{v}^{K}+f_{w}^{K}=2 f_{v}^{K} .
$$

But $f^{K}$ tends to $f$, the density of $S$, and $f_{v}^{K}(t)$ tends to $e^{-t}$ by Proposition 7 , so $f(t) \leqslant 2 e^{-t}$ and in particular $f(0) \leqslant 2$. Now by Lemma $11 G(t)=P(S>t)$ is a completely monotone function, which easily implies

Thus

$$
\begin{gathered}
G(t) \geqslant e^{-t \beta}, \quad \text { where } \quad \beta=-G^{\prime}(0) \leqslant 2 . \\
\mathbb{E} S=\int_{0}^{\infty} G(t) d t \geqslant \int_{0}^{\infty} e^{-2 t} d t=\frac{1}{2},
\end{gathered}
$$

establishing (18). Next, consider computing $\mathbb{E}_{n} T_{w}$ by conditioning on $T$ :

$$
\mathbb{E}_{n} T_{w}=\mathbb{E}_{n} T+P_{\pi}\left(T_{v}<T_{w}\right) \mathbb{E}_{v} T_{w}=\mathbb{E}_{n} T+\frac{1}{2} \mathbb{E}_{v} T_{w}
$$


by Proposition 3. Rearranging,

$$
\mathbb{E}_{v} T_{w} / \alpha=2\left(1-\mathbb{E}_{\pi} T / \alpha\right) .
$$

Using (18), we have limsup $\mathbb{E}_{v} T_{w} / \alpha \leqslant 1$. But trivially $\max _{v, w} \mathbb{E}_{v} T_{w} / \alpha \geqslant 1$ for each $K$, so we have established

$$
\max _{v, w} \mathbb{E}_{v} T_{w} / \alpha \rightarrow 1,
$$

which is the first assertion of Proposition 8.

Now we shall prove the second assertion in the special case $\theta=1$, that is when $\mathbb{E}_{v} T_{w} / \alpha \rightarrow 1$. We use the argument above in the reverse direction. By $(20), \mathbb{E}_{n} T / \alpha \rightarrow \frac{1}{2}$. Thus if $S$ is a subsequential weak limit of $(T / \alpha)$ then $\mathbb{E} S=\frac{1}{2}$ and so the inequality in (19) must be an equality: $P(S>t)=e^{-2 t}$. This shows that

$$
T / \alpha \rightarrow \mu_{2}
$$

where $\mu_{2}$ denotes the exponential distribution with mean $\frac{1}{2}$. Now consider the walk started with the uniform distribution $\pi$, and calculate the distribution of $T_{w_{K}}$ by conditioning on $T^{K}=\min \left(T_{v_{K}}, T_{w_{K}}\right)$. Using Proposition 3,

$$
T_{w_{K}}=T^{K}+B_{K} I_{K}
$$

where

(i) $B_{K}$ has distribution $\mathscr{L}_{v_{K}}\left(T_{w_{K}}\right)$,

(ii) $P\left(I_{K}=1\right)=P\left(I_{K}=0\right)=\frac{1}{2}$,

(iii) $T^{K}, B_{K}$ and $I_{K}$ are independent.

But we know $\mathscr{L}\left(T_{w_{K}} / \alpha\right) \rightarrow \mu_{1}$ and $\mathscr{L}\left(T^{K} / \alpha\right) \rightarrow \mu_{2}$, and then by considering transforms in the identity (21) we deduce that $\mathscr{L}\left(B_{K} / \alpha\right) \rightarrow \mu_{1}$. This is assertion $(d)$ of the Proposition, in the special case $\theta=1$.

For the general case we need a lemma, whose proof is deferred.

Lemma 14. For any subset $A$ of vertices in a vertex-transitive graph,

$$
\max _{v} \mathbb{E}_{v} T_{A} \leqslant\left(\left|A^{c}\right| /|A|\right) \max _{v, w} \mathbb{E}_{v} T_{w}
$$

Let $v=v_{K}, w=w_{K}$ be such that

$$
\mathbb{E}_{v} T_{w} / \mathbb{E}_{n} T_{w} \rightarrow \theta .
$$

Consider sets of vertices of the form

$$
A^{K}=\left\{a \in V_{K}: \mathbb{E}_{a} T_{w} \geqslant\left(1-\epsilon_{K}\right) \mathbb{E}_{\pi} T_{w}\right\} .
$$

On choosing $\epsilon_{K}$ tending to 0 sufficiently slowly, assertion $(c)$ of the proposition implies $\left|A^{K}\right| /\left|V_{K}\right| \rightarrow 1$, and then Lemma 14 implies

$$
\mathbb{E}_{v}\left(T_{A} / \alpha\right) \rightarrow 0 \text {. }
$$

Now consider $\theta_{K}=P\left(T_{w_{K}}>T_{A} K\right)$. By conditioning on this latter event,

$$
\mathbb{E}_{v} T_{w} / \alpha=\mathbb{E}_{v} \min \left(T_{w}, T_{A}\right) / \alpha+\theta_{K} \mathbb{E}_{\rho} T_{w} / \alpha,
$$

where $\rho=\rho_{K}$ is the distribution of $X\left(T_{A}\right)$ given $T_{A}<T_{w}$. From the definition of $A_{K}$ and the special case already proved,

$$
\mathbb{E}_{\rho} T_{w} / \alpha \rightarrow 1, \quad \mathscr{L}_{\rho}\left(T_{w} / \alpha\right) \rightarrow \mu_{1} .
$$


The hypothesis of part $(d)$ of the proposition is $\mathbb{E}_{v} T_{w} / \alpha \rightarrow \theta$, so using (22) and (23) we see that $\theta_{K} \rightarrow \theta$. The same argument, applied to distributions rather than expectations, shows that

$$
\mathscr{L}_{v}\left(T_{w} / \alpha\right) \rightarrow \theta \mu_{1}+(1-\theta) \delta_{0}
$$

the desired conclusion.

Proof of Lemma 14.

$$
\begin{aligned}
|V| \mathbb{E}_{n} T_{v}= & \sum_{w} \mathbb{E}_{w} T_{v} \\
= & \sum_{w} \mathbb{E}_{v} T_{w} \text { by Proposition } 3 \\
& \geqslant \sum_{w \in A} \mathbb{E}_{v} T_{w} \\
& =\sum_{w \in A}\left(\mathbb{E}_{v} T_{A}+\mathbb{E}_{\rho} T_{w}\right) \\
& \text { where } \rho \text { is the first hitting place distribution on } A \\
= & |A| \mathbb{E}_{v} T_{A}+\sum_{w \in A} \mathbb{E}_{\rho} T_{w} \\
= & |A| \mathbb{E}_{v} T_{A}+\sum_{w} \mathbb{E}_{\rho} T_{w}-\underset{w \in A^{c}}{\sum_{\rho} \mathbb{E}_{p} T_{w}} \\
& \geqslant|A| \mathbb{E}_{v} T_{A}+|V| \mathbb{E}_{n} T_{w}-\left|A^{c}\right| \max _{v, w} \mathbb{E}_{v} T_{w},
\end{aligned}
$$

where the middle term is obtained by considering the $\rho$-average over $v$ in (24). The result follows on rearranging.

Remark. Proposition 8 has an extension from singletons to $L$-tuples. Suppose

$$
\left(w_{0}, w_{1}, \ldots, w_{L}\right)=\left(w_{K, 0}, w_{K, 1}, \ldots, w_{K, L}\right) \in V_{K},
$$

where $L$ is fixed. Suppose

Then

$$
\mathbb{E}_{w_{i}} T_{w_{l}} / \alpha \rightarrow 1 \text { for all } i \neq l .
$$

where the $\left(\xi_{i}\right)$ are independent with exponential(1) distribution. This can be proved using the same argument as in the 'special case' above. The general case where (26) fails is treated heuristically in [3], section B 12 .

\section{Coefficient of variation}

We first discuss the significance of Proposition 6 , and then give its proof. Let $M$ be a set of continuous-time Markov chains. Let $T(M)$ be the set distributions $\mathscr{L}(a T)$, where $a>0$ is constant and $T$ is a first passage time between some pair of states for some chain in $M$. Let $\bar{T}(M)$ be the closure of $T(M)$, under convergence in distribution. It is classical that

$$
\bar{T}(\text { all Markov chains })=(\text { all distributions on }[0, \infty)) .
$$


See [6] for a modern account. It is natural to ask how far the class of chains can be restricted without affecting this result. A slightly complicated construction [4] shows

$$
\bar{T} \text { (random walks on regular graphs) }=(\text { all distributions on }[0, \infty)) \text {. }
$$

On the other hand, Proposition 6 suggests (and it is not hard to verify formally) that $\bar{T}$ (random walks on vertex-transitive graphs) does not contain all distributions: there is a lower bound for the coefficient of variation in this set.

Problem 15. What is $\bar{T}$ (random walks on vertex-transitive graphs)?

This is perhaps difficult. In view of (5) it is related to the problem of finding all possible rescaled limits of functions

$$
p_{v w}(t)=P_{v}(X(t)=w)
$$

for sequences of vertex-transitive graphs.

Proposition 6 will be deduced from the following more general bound.

Proposition 16. Consider a continuous-time reversible Markov chain which starts at a state $i$. Let $A$ be a subset of states with $i \in A^{c}$. Let $T$ be the time of first return to $i$ after hitting $A$ :

Then

$$
\begin{gathered}
T=\min \left\{t>T_{A}: X(t)=i\right\} . \\
\frac{\operatorname{var} T}{(\mathbb{E} T)^{2}} \geqslant \frac{e-2}{2 e-2} .
\end{gathered}
$$

Given Proposition 16, the proof of Proposition 6 is almost obvious. Consider a continuous-time random walk on a vertex-transitive graph started at $v$. Write $T_{w}$ for the first hitting time on $w$ and $T_{w}+U_{v}$ for the time of the subsequent hit on $v$. Then Proposition 16 can be applied to $T=T_{w}+U_{v}$. But $T_{w}$ and $U_{v}$ are independent and, by Proposition 2, have the same distribution. Thus

$$
\frac{\operatorname{var}_{v} T_{w}}{\left(\mathbb{E}_{v} T_{w}\right)^{2}}=2 \frac{\operatorname{var} T}{(\mathbb{E} T)^{2}} \geqslant \frac{e-2}{e-1}
$$

This is the conclusion of Proposition 6 for continuous-time walks. Relation (8) now yields the stated form of the Proposition in the discrete-time setting.

Remark. For general continuous-time reversible chains, the argument above shows that, if $\mathscr{L}_{v} T_{w}$ is almost constant (i.e. has small coefficient of variation) then $\mathbb{E}_{w} T_{v} / \mathbb{E}_{v} T_{w}$ must be large.

Proof of Proposition 16. Let $T_{i}^{+}$be the first return time to $i$. Write

$$
p=P\left(T_{i}^{+}<T_{A}\right), \quad q=1-p=P\left(T_{A}<T_{i}^{+}\right),
$$

and suppose $R^{\prime}$ has the conditional distribution $\mathscr{L}\left(T_{i}^{+} \mid T_{i}^{+}<T_{A}\right), S^{\prime}$ has the conditional distribution $\mathscr{L}\left(T_{\imath}^{+} \mid T_{t}^{+}>T_{A}\right.$ ) (recall we are assuming that the chain starts at $i$ ). The distribution of $T_{i}^{+}$is a mixture of the distributions of $R^{\prime}$ and $S^{\prime}$ :

$$
\mathscr{L}\left(T_{i}^{+}\right)=p \mathscr{L}\left(R^{\prime}\right)+q \mathscr{L}\left(S^{\prime}\right)
$$

and so in particular

$$
\mathbb{E} T_{i}^{+}=p \mathbb{E} R^{\prime}+q \mathbb{E} S^{\prime}
$$




$$
\operatorname{var} T_{i}^{+}=p \operatorname{var} R^{\prime}+q \operatorname{var} S^{\prime}+\left(\mathbb{E} R^{\prime}-\mathbb{E} S^{\prime}\right)^{2} p q
$$

Now the time interval $[0, T]$ consists of a geometric number $N$ of returns to $i$ which avoid $A$, followed by one return after hitting $A$. So

$$
T=R_{1}^{\prime}+\ldots+R_{N}^{\prime}+S^{\prime}
$$

where $R_{i}^{\prime}$ has the distribution of $R^{\prime}, P(N=n)=p^{n} q(n \geqslant 0)$ and these random variables are independent of each other and $S^{\prime}$.

Relations (28)-(30) allow us to express the mean and variance of $T$ in terms of the underlying quantities $p, R^{\prime}$, etc. After some algebra we get

$$
\begin{gathered}
\mathbb{E} T=q^{-1} \mathbb{E} T_{i}^{+}, \\
\operatorname{var} T=q^{-1} \operatorname{var} T_{i}^{+}+p q^{-2}\left(2\left(\mathbb{E} T_{i}^{+}\right) \mathbb{E} R^{\prime}-\left(\mathbb{E} T_{i}^{+}\right)^{2}\right) .
\end{gathered}
$$

The chain started at $i$ holds in $i$ for an exponential time $\xi$, independent of its subsequent behaviour, before its first jump. So we can write

$$
R^{\prime}=\xi+R, \quad S^{\prime}=\xi+S, \quad T_{i}^{+}=\xi+Z .
$$

By scaling time, we may assume $\mathbb{E} \xi=1$. Note that Lemma 11 implies that $Z$ has a CM distribution. Write $a=\mathbb{E} Z$. Substituting into the equations above and doing straightforward calculations gives

$$
\frac{\operatorname{var} T}{(\mathbb{E} T)^{2}}=\frac{1+q \operatorname{var} Z+p\left((2+2 a) \mathbb{E} R-a^{2}\right)}{(1+a)^{2}}
$$

Now consider $p$ and $\mathscr{L}(Z)$ fixed. Then $R$ satisfies the 'mixture' constraint implied by (27):

$$
\mathscr{L}(Z) \geqslant p \mathscr{L}(R) \text {. }
$$

Clearly, subject to this constraint $\mathbb{E} R$ is smallest when $R$ has the distribution of $Z$ given $Z \leqslant t$, for $t$ defined by $P(Z \leqslant t)=p$. So for this $t$,

$$
\mathbb{E} R \geqslant \mathbb{E}(Z \mid Z \leqslant t)=p^{-1} \mathbb{E} Z 1_{(Z \leqslant t)}
$$

Now varying $p$, or equivalently varying $t$, gives

$$
\frac{\operatorname{var} T}{(\mathbb{E} T)^{2}} \geqslant \inf _{t>0} \frac{1+P(Z>t) \operatorname{var} Z+(2+2 a) \mathbb{E} Z 1_{(Z \leqslant t)}-a^{2} P(Z \leqslant t)}{(1+a)^{2}} .
$$

The numerator may be rewritten as $1+2 \mathbb{E} Z 1_{(Z \leqslant t)}+a^{2}+h(t)$ where

$$
h(t)=P(Z>t) \mathbb{E} Z^{2}-2 a \mathbb{E} Z 1_{(Z>t)} .
$$

By calculus this is minimized at $t_{0}=\left(\mathbb{E} Z^{2}\right) /(2 a)$. So

$$
h(t) \geqslant h\left(t_{0}\right)=-\mathbb{E}\left[2 a Z-\mathbb{E} Z^{2}\right]^{+} \geqslant-2 a^{2} / e
$$

by Lemma 17 below. Substituting into (31),

$$
\frac{\operatorname{var} T}{(\mathbb{E} T)^{2}} \geqslant \frac{1+a^{2}(1-2 / e)}{(1+a)^{2}} .
$$

Minimizing over $a>0$ gives minimum value $(e-2) /(2 e-2)$, establishing the proposition. 
Lemma 17. Suppose that $X$ has a CM distribution, $\mathbb{E} X=a, \mathbb{E} X^{2}=s^{2}$. Then

$$
\mathbb{E}\left(2 a X-s^{2}\right)^{+} \leqslant 2 a^{2} / e .
$$

Proof. If $\xi$ has exponential(1) distribution and $b, c>0$ then by an easy calculation $\mathbb{E}(b \xi-c)^{+}=b e^{-c / b}$. Write $X=V \xi$, so that $\mathbb{E} V=a, \mathbb{E} V^{2}=\frac{1}{2} s^{2}$. Then

$$
\begin{aligned}
\mathbb{E}\left(2 a X-s^{2}\right)^{+} & =\mathbb{E}\left(2 a V \xi-s^{2}\right)^{+} \\
& =\mathbb{E}\left(2 a V e^{-\delta^{2} /(2 a V)}\right) \quad \text { by conditioning on } V \\
& =\mathbb{E}\left(4 a^{2} V^{2} / s^{2}\right)\left(s^{2} /(2 a V)\right) e^{-s^{2} / 2 a V} \\
& \leqslant \mathbb{E}\left(4 a^{2} V^{2} / s^{2}\right) e^{-1} \quad \text { because } x e^{-x} \leqslant e^{-1} \\
& =2 a^{2} e^{-1}
\end{aligned}
$$

The author's research is supported by NSF grant MCS84-03239.

\section{REFERENCES}

[1] D. J. Aldous. An introduction to covering problems for random walks on graphs. J. Theoret. Probab. 2 (1989), 87-89.

[2] D. J. Aldous. Markov chains with almost exponential hitting times. Stochastic Process. Appl. 13 (1982), 305-310.

[3] D. J. Aldous. Probability Approximations via the Poisson Clumping Heuristic (SpringerVerlag, 1989).

[4] D. J. ALbous. Random walks on large graphs. (Unpublished lecture notes, projected monograph.)

[5] R. Aleliunas et al. Random walks, universal traversal sequences, and the complexity of maze traversal. In Proc. 20th IEEE Found. Comp. Sci. Symp. (IEEE, 1979), pp. 218-233.

[6] S. Asmussen. Applied Probability and Queues (Wiley, 1987).

[7] N. Biggs. Algebraic Graph Theory (Cambridge University Press, 1974).

[8] A. Broder and A. R. Karlin. Bounds on the cover time. J. Theoret. Probab. 2 (1989), 101-120.

[9] J. T. Cox. Coalescing random walks and voter model consensus times on the torus. Ann. Probab. (to appear).

[10] P. Diaconis. Group Theory in Statistics (Institute of Mathematical Statistics, Hayward CA, 1988).

[11] P. G. Doyle and J. L. SNell. Random Walks and Electrical Networks (Mathematical Association of America, 1984).

[12] L. Flatto, A. M. Odlyzko and D. B. Wales. Random shuffles and group representations. Ann. Probab. 13 (1985), 154-178.

[13] F. Gobel and A. A. JAGers. Random walks on graphs. Stochastic Process. Appl. 2 (1974), 311-336.

[14] J. Keilson. Markov Chain Models - Rarity and Exponentiality (Springer-Verlag, 1979).

[15] J. G. Kemeny and J. L. Snell. Finite Markov Chains (Van Nostrand, 1969).

[16] G. Letac. Les fonctions spheriques d'un couple de Gelfand symmetrique et les chaines de Markov. Adv. in Appl. Probab. 14 (1982), 272-294.

[17] J. W. Pitman. Occupation measures for Markov chains. Adv. in Appl. Probab. 9 (1977), 69-86.

[18] L. Takacs. Random flights on regular graphs. Adv. in Appl. Probab. 16 (1984), 618-637.

[19] A. R. D. vas SLIJPE. Random walks on the triangular prism and other vertex-transitive graphs. J. Comput. Appl. Math. 15 (1986), 383-394.

[20] N.T. VA Ropoclos. Isoperimetric inequalities and Markov chains. J. Funct. Anal. 63 (1985). 215-239.

An extensive bibliography concerning random walks on graphs is available from the author. 